\title{
Monte Carlo Simulation of Carrier Capture at Deep Centers for Silicon and Gallium Arsenide Devices
}

\author{
A. Palma, J.A. Jiménez-Tejada, A. Godoy and J.E. Carceller \\ Departamento de Electrónica y Tecnologia de Computadores. Facultad de Ciencias. \\ Universidad de Granada. 18071 Granada, SPAIN
}

\begin{abstract}
This work provides a direct way to include multiphonon capture at deep levels in the simulation of electron transport by the Monte Carlo method. This has been possible by adding the capture probability as one more scattering probability together with lattice mechanisms. To check this probability, numerical capture cross sections for deep centers in $\mathrm{Si}$ and GaAs obtained by our method have been fitted with experimental measurements.
\end{abstract}

\section{Introduction}

The multiphonon-emission process, responsible for carrier capture in bulk deep center$\mathrm{s}$, has been included in the framework of a standard Monte Carlo simulation [1] of the electron transport in $\mathrm{Si}$ and $\mathrm{GaAs}$ semiconductors doped with deep neutral impurities. Multiphonon-emission probability has been added to the simulation lattice-scattering mechanisms. This work comprises a first step in the implementation of this process in device simulators.

At the limit of low temperatures and weak coupling, the probability of emission of p phonons with energy $\hbar \omega, \mathrm{W}^{\mathrm{mph}}$, has been calculated on the basis of the adiabatic formalism developed by Ridley $[2,3]$ :

$$
W^{m p h}=\frac{16 \pi^{2} \omega S^{p} e^{-S}\left(\nu_{T} a^{*}\right)^{3}}{V(2 \pi p)^{1 / 2}(p / e)^{p}} p[0.4+0.18(p-1)](n+1)^{p} e^{-2 n S},
$$

where $\mathrm{S}$ is the Huang-Rhys factor $(\mathrm{S}<<\mathrm{p}), \mathrm{V}$ the crystal volume, and $\mathrm{a}^{*}$ the effective Bohr radius in the semiconductor. The delta function model has been used to model the center bound state, $\phi_{T}(r) \propto r^{-1} e^{-r / \nu_{T} a^{*}}$. Dependence with the deep energy level, $\mathrm{E}_{\mathrm{T}}$, is through $\nu_{\mathrm{T}}$ as follows:

$$
\nu_{T}^{2}=\frac{E_{H}^{*}}{E_{T}}
$$

where $\mathrm{E}_{\mathrm{H}}^{*}$ is the effective Rydberg energy (32 meV in $\mathrm{Si}$ and $5.3 \mathrm{meV}$ in GaAs), and dependence with the temperature $\mathrm{T}$ is included in the occupation factor of phonons, 
$n=\left(e^{\hbar \omega / k_{B} T}-1\right)^{-1}$, where $\mathrm{k}_{\mathrm{B}}$ is the Boltzmman constant. In this eq., we considered the emitted phonons to be the non-polar LO phonons $(\hbar \omega=63 \mathrm{meV})$ in $\mathrm{Si}$ and $(\hbar \omega$ $=30 \mathrm{meV}$ ) in GaAs. Therefore, the Huang-Rhys factor is the only free parameter in Expression 1. In order to verify the capture probability, in the next section we have numerically calculated the thermal dependence of the capture cross sections for several deep centers in $\mathrm{Si}$ and in GaAs.

\section{Numerical procedure}

The task of incorporating this probability in the Monte Carlo simulation was solved by restricting the carrier motion to one impurity space. Therefore, in a sample with an empty impurity concentration of $\mathrm{N}_{T}$, the real probabilities included were:

$$
<W^{m p h}>=W^{m p h} V N_{T},
$$

which means that we are simulating the motion of one electron in the average volume corresponding to one trap. The third body [3] and the screening effects were neglected.

In the Monte Carlo simulation, all phonon mechanisms were included and the nonparabolicity effects were accounted for both in $\mathrm{Si}$ and in GaAs. The numerical procedure was as follows: One electron is introduced with the thermal energy corresponding to the lattice temperature and is allowed to move without including for the multiphonon process in order to avoid dependencies with the initial carrier state. After a. certain number of scatterings (15000), the multiphonon mechanism is activated. At precisely the moment when this mechanism is stochastically chosen, the electron is considered captured, and the mean velocity and the time spent since the multiphonon probability inclusion, the so-called capture time, are recorded. This one-electron procedure is repeated for a very large number of carriers, and the average values of the mean velocities, $\langle v\rangle$, and the capture times, $\left\langle\tau_{c}\right\rangle$, are used to calculate the average thermal capture cross section for electrons, $\sigma_{n}$ :

$$
\sigma_{n}=\frac{1}{N_{T}<v><\tau_{c}>}
$$

\section{Results}

In order to check whether the average capture time is the proper value to use in Eq. 4 , the distribution of electrons, $\mathrm{N}_{e}$, with the capture-lime interval is plotted is Fig. 1 . This figure shows one example of the exponentiality of this numerical magnitude as the Schokley-Read-Hall statistic predicts. We have fitted our results with experimental data $[4,5]$ of capture cross sections vs. temperalure for the acceptor level of $\mathrm{Au}$ in silicon (Fig.2) and the A defect in gallium arsenide (Fig.3). The Huang-Rhys factors obtained in these fittings agree with theoretical and experimental measurements $[2,6]$.

In summary, the inclusion of the capture mechanism with the rest of the lattice mechanisms allows a better understanding of this phenomena, showing as it does the relationship between the multiphonon process and the scattering mechanisms. The agreement between our results and experimental data has been achieved with physical values of the different parameters of Eq. 1, resulting in an easy way of implementing this capture mechanism in carrier transport simulators. 


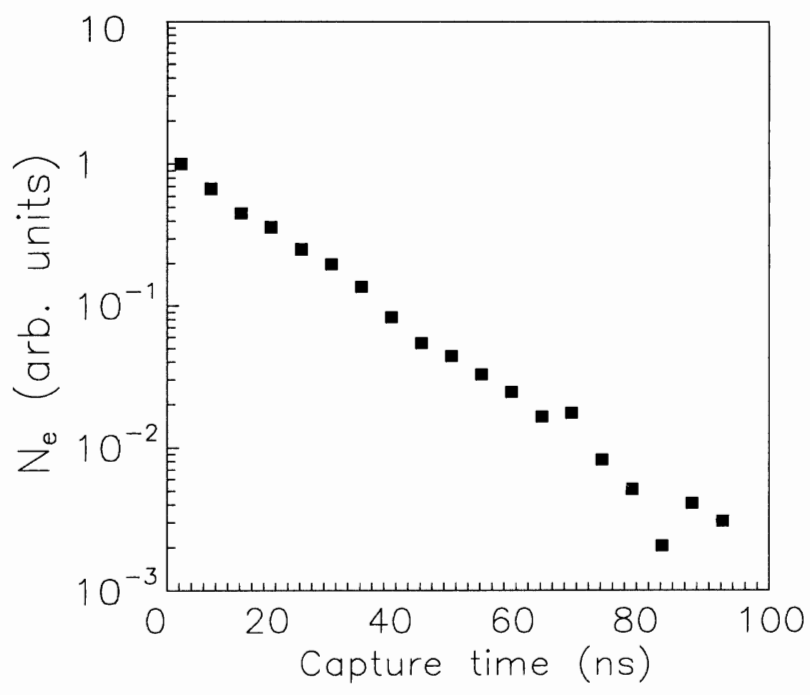

Figure 1: Histogram of $\mathrm{N}_{e}$ vs. capture time. Simulation for $\mathrm{Pt}$ acceptor level in $\mathrm{Si}$ with $\mathrm{N}_{\mathrm{T}}=5 \cdot 10^{14} \mathrm{~cm}^{-3}$ at $\mathrm{T}=80 \mathrm{~K}$

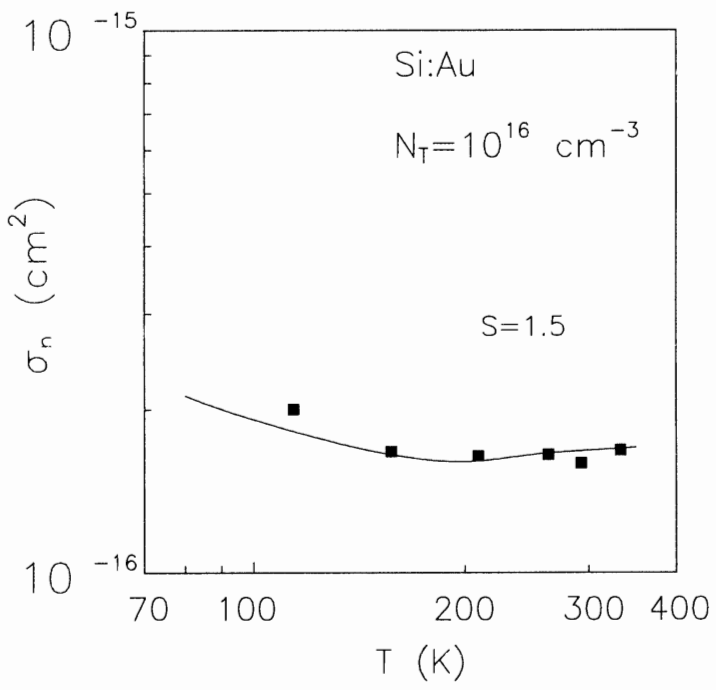

Figure 2: Comparison between experimental[4] (symbols) and numerical (line) $\sigma_{n}$ of electrons for Au acceptor level in Si 


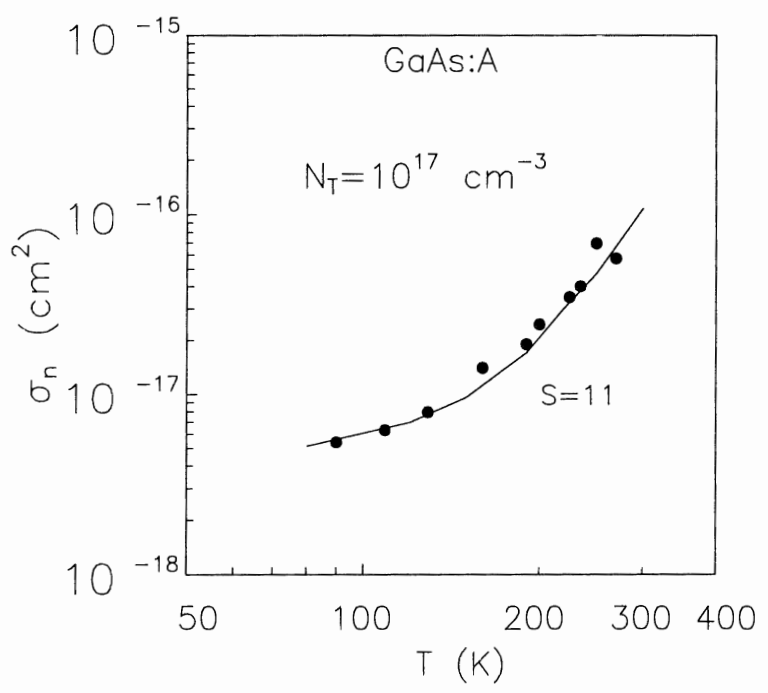

Figure 3: Experimental[5] (symbols) and numerical (line) $\sigma_{n}$ for A center in GaAs

\section{References}

[1] C. Jacoboni and L. Reggiani, "The Monte Carlo method for the solution of charge transport in semiconductors with applications to covalent materials," Rev. Mod. Phys, vol. 55, no. 3, pp. 645-705, 1983.

[2] B.K. Ridley, Quantum Processes in Semiconductors, Clarendon Press, Oxford. 1993

[3] B.K. Ridley, "On the multiphonon capture rate in semiconductors," Solid-State Electron., vol 21, pp. 1319-1323, 1978

[4] D.V. Lang, H.G. Grimmeiss, E. Meijer and M. Jaros, "Complex nature of goldrelated deep levels in silicon," Phys. Rev. B, vol. 22, no 7, pp. 3917-3934, 1980

[5] C.H. Henry and D.V. Lang, "Nonradiative capture and recombination by multiphonon emission in GaAs and GaP," Phys. Rev. B, vol. 15, no. 2, pp. 989-1016, 1977

[6] S.T. Pantelides, Deep Centers in Semiconductors, Gordon and Breach Science Publishers, New York, 1992 